\title{
IMPORTANCIA DE LA ENFERMERA ESCOLAR SEGÚN LA PERCEPCIÓN DE FUNCIONARIOS DE COLEGIOS BÁSICOS DE UNA PROVINCIA DE CHILE
}

\author{
IMPORTANCE OF THE SCHOOL NURSE ACCORDING TO THE \\ PERCEPTION OF OFFICIALS OF BASIC SCHOOLS OF A PROVINCE OF \\ CHILE
}

\section{IMPORTÂNCIA DA ENFERMAGEM ESCOLAR DE ACORDO COM A PERCEPÇÃO DE FUNCIONÁRIOS DE ESCOLAS BÁSICAS DE UMA PROVÍNCIA DO CHILE}

\author{
Bania Catalina Ceballo Mella* \\ Daicy Marioly Fonseca Sanhueza** \\ Naibeth Scarlette Fuenzalida Palma*** \\ Lidia Lisette Morales RodríGuez ${ }^{* * * *}$ \\ Darynka Angélica Parada Jiménez***** \\ Ismael Antonio Morales Ojeda******
}

\section{RESUMEN}

Objetivo: Describir la importancia de la enfermera escolar según la percepción de los funcionarios de colegios básicos de una provincia al sur de Chile. Material y Método: Estudio cuantitativo, descriptivo, transversal que, a partir de un universo de 169 funcionarios de 5 colegios públicos y privados, obtuvo una muestra de $126(75 \%)$ personas quienes, posterior a dar su consentimiento informado, respondieron el instrumento "Percepción de la importancia de la enfermera escolar", desde la perspectiva de sus funciones asistenciales, educativas, investigativas, administrativas y de gestión. La investigación fue aprobada por el Comité de ética de la Universidad Adventista de Chile. Resultados: El 94\% de los funcionarios refieren que es importante la enfermera escolar para actuar en emergencias ocurridas durante el horario escolar; un 93,5\% percibe que la enfermera es indispensable para atender en primeros auxilios; un 92,25\% cree que los padres estarán tranquilos al saber que hay un profesional de salud en la escuela y un 91,75\% dice que el profesional de enfermería puede educar al alumnado sobre infecciones de transmisión sexual. Conclusión: Según los funcionarios de los colegios, la presencia de una enfermera escolar es importante y debiera estar presente para mantener la salud de los estudiantes.

Palabras clave: Enfermera/o Escolar; Escolares; Profesores de educación general básica; Funcionario administrativo.

\footnotetext{
* al ***** Licenciada en Enfermería, Universidad Adventista de Chile, Chillán, Chile.

*** Autora de correspondencia, Email: importanciaenfermeraescolar@gmail.com.

****** Doctor en Ciencias Biomédicas, Universidad Adventista de Chile, Chillán, Chile. Email: publicacionesdirinves@gmail.com
} 


\begin{abstract}
Objective: To describe the importance of the school nurse as perceived by school staff members of elementary schools from a province south Chile. Materials and Methods: Quantitative, descriptive, cross-sectional study, using a sample of $126(75 \%)$ participants from a population of 169 school staff members of 5 public and private schools. After giving informed consent, the participants answered the instrument "Perception of the importance of the school nurse", from the perspective of their caregiving, educational, research and management functions. The research was approved by the Ethics Committee of the Chilean Adventist University. Results: 94\% of school staff members indicate that the school nurse is important when acting in emergencies during school hours; $93.5 \%$ consider that the school nurse is vital when it comes to providing first aid; $92.25 \%$ believe that parents feel reassured when there is a health professional in the school, and $91.75 \%$ say that nurses can educate students on sexually transmitted infections. Conclusion: According to school staff members, the presence of a school nurse is important and should be promoted for maintaining student's health.
\end{abstract}

Keywords: School nurse; Students; Elementary school teachers; School staff members.

\title{
RESUMO
}

Objetivo: Descrever a importância da enfermeira escolar de acordo com a percepção dos funcionários da escola básica de uma província sul do Chile. Material e Método: Estudo quantitativo, descritivo, transversal que, de um universo de 169 funcionários de 5 escolas públicas e privadas, obteve uma amostra de 126 (75\%) pessoas que, depois de dar o consentimento informado, responderam ao instrumento "Percepção da importância da enfermeira escolar", na perspectiva de suas funçóes assistenciais, educacionais, de pesquisa, administrativas e gerenciais. A pesquisa foi aprovada pelo Comitê de Ética da Universidade Adventista do Chile. Resultados: $94 \%$ dos funcionários relata que a enfermeira escolar é importante para atuar em emergências durante o horário escolar; $93,5 \%$ percebe que a enfermeira é indispensável para o atendimento de primeiros socorros; 92,25\% acredita que os pais ficam calmos sabendo que existe um profissional de saúde na escola e 91,75\% diz que o profissional de enfermagem pode educar os alunos sobre infecçôes sexualmente transmissíveis. Conclusão: Segundo os funcionários da escola, a presença de uma enfermeira escolar é importante e deve estar presente para manter a saúde dos alunos.

Palavras-chave: Enfermeira escolar; Escolares; Professores do Ensino Básico Geral; Oficial administrativo.

Fecha de recepción: 28/08/2019

Fecha de aceptación: 13/12/2019

\section{INTRODUCCIÓN}

La ausencia de un profesional para la atención de la salud escolar en los establecimientos educacionales, actualmente asumida por paradocentes, arriesga problemas o daños futuros en la salud de los alumnos. En Chile los escolares y preescolares pasan la mayor parte de su tiempo en la escuela aprendiendo distintas habilidades, lo que incluye también la realización de actividades como recreación y deporte, eventos donde es inevitable el riesgo de sufrir algún accidente ${ }^{(1,2)}$. De ser así y ocurrir algún imprevisto, se precisa de una actuación rápida y correcta por parte de un profesional de la salud que tenga experticia en primeros auxilios.

Según Martín ${ }^{(2)}$, la educación impartida en el ámbito escolar al personal docente, respecto a primeros auxilios, no se realiza con la misma relevancia y capacidades como lo realiza un profesional de enfermería. En el contexto actual la mayoría de los colegios no disponen de un profesional del área de la salud que pueda asistir a los estudiantes en caso de lesiones o accidentes, rol que es asumido por docentes del mismo 
establecimiento, a pesar de que al caracterizar las consultas por accidentes escolares, el diagnóstico más frecuente corresponde a lesiones de muñeca y mano: luxaciones, esguinces y desgarros ${ }^{(3)}$, incidentes que podrían comenzar a tratarse, tempranamente, en el lugar del hecho, por una persona capacitada.

Generalmente los profesores manifiestan un gran compromiso al cuidar de los nińos, sin embargo, no poseen las competencias necesarias para actuar en situaciones de emergencia, de ahí que es necesario incluir en las escuelas a la enfermera escolar, no solo para ayudar en la emergencia, sino también para educar al cuerpo de profesionales y administrativos a estar preparados frente a los casos de urgencia. De ahí la necesidad del rol de la enfermera en la escuela.

La presencia de este profesional a nivel escolar no es una novedad pues hay algunas instituciones que ya cuentan con su participación ${ }^{(4-6)}$, sin embargo, la mayoría de los colegios en el país no tienen a este profesional como parte de sus trabajadores ${ }^{(7,8)}$.

La enfermera, en el ámbito escolar, brinda los cuidados de salud necesarios en la comunidad estudiantil y posee competencias que acreditan sus conocimientos y habilidades dirigidas a contribuir con el bienestar físico, mental y social de dicha comunidad, desarrollando labores de promoción de la salud y prevención de las enfermedades, adelantándose a los sucesos desfavorables y actuando eficazmente sobre ellos, por lo que debe estar presente en la institución educacional durante todo el horario escolar ${ }^{(9)}$. Particularmente en Chile ya existen antecedentes que definen la actuación de la enfermera escolar que incluyen las acciones y responsabilidades del cargo. Algunos ejemplos de establecimientos educacionales en Chile que cuentan permanentemente con este profesional son: Colegio Instituto San José(10) de Cañete, Colegio Leonardo Da Vinci ${ }^{(1)}$ de Las Condes y el Colegio Saint Margaret's S.A. ${ }^{(12)}$, lugares donde se cuenta con protocolos para el uso adecuado de la sala de enfermería, de procedimientos y de derivación en caso de requerir una atención de salud más especializada ${ }^{(13)}$. El organismo que respalda su actuar es la Sociedad Chilena de Enfermería en Salud Escolar (SOCHIESE) y define sus funciones en los niveles pre-básicos, básicos, medios y superior, contribuyendo con ello al adecuado manejo del alumnado ${ }^{(14)}$. La enfermería escolar también tiene otras funciones: actúa en caso de enfermedades crónicas de los escolares, gestiona la prestación de servicios de salud, posibilita la realización de exámenes a los estudiantes para asegurar la mantención y prevención de enfermedades, vacuna según la edad correspondiente, educa en prevención de drogas y hábitos saludables ${ }^{(15)}$ y tiene un importante rol en la prevención del bullying y la educación sexual, entre otros. Enfermería, como profesión, cumple el rol de enlace entre alumnos, padres/apoderados, todo el personal del establecimiento y el equipo de salud ${ }^{(16-18)}$ y proporciona educación y material didáctico orientado al profesorado y al alumnado respecto a primeros auxilios ${ }^{(19)}$.

Según lo descrito, este trabajo buscó indagar la percepción de funcionarios de colegios básicos respecto de la importancia de la enfermera escolar e identificar las funciones más relevantes, desde su perspectiva.

\section{MATERIALES Y MÉTODOS}

Tipo de estudio: El estudio tuvo un abordaje cuantitativo, de tipo descriptivo y transversal, desarrollado entre los meses de agosto y octubre del año 2018.

Unidad de análisis: Estuvo constituida por profesores de educación general básicay funcionarios o administrativos que trabajan en colegios públicos y privados del sector Las Mariposas, Provincia de Diguillín, Región de Nuble, Chile. Una vez recibida la aprobación del Comité de Ética de la Universidad Adventista de Chile, se gestionó la asociación colaborativa entregando las solicitudes a todos los establecimientos señalados $\mathrm{y}$, una vez obtenida su autorización, se comenzó con el proceso y la aplicación de la encuesta. Todos los participantes dieron su consentimiento informado que explicitaba el carácter anónimo y confidencial de los datos.

Universo y muestra: A partir de un universo de 169 personas, se obtuvo una muestra probabilística de 126 funcionarios (Tabla 1). Como criterio de inclusión se consideró el estar presente en el establecimiento al momento de aplicar la encuesta y la exclusión se aplicó a aquellos funcionarios que no completaron todas las preguntas del instrumento. 
Tabla 1. Distribución de universo y muestra según establecimientos educacionales, Sector Las Mariposas, Comuna de Chillán-Chile, 2018 ( $\mathrm{n}=126)$.

\begin{tabular}{lccc}
\hline Establecimientos Educacionales & Universo (N) & Muestra (n) & $\mathbf{\%}$ \\
\hline Colegio Alcázares de Nuble & 33 & 18 & $55 \%$ \\
Colegio Ciudad Educativa. & 52 & 41 & $79 \%$ \\
Colegio Adventista Las Mariposas & 32 & 25 & $78 \%$ \\
Escuela Especial de Lenguaje My College & 33 & 30 & $91 \%$ \\
Colegio Camilo Henríquez G-283 & 19 & 12 & $63 \%$ \\
\hline TOTAL & $\mathbf{1 6 9}$ & $\mathbf{1 2 6}$ & $\mathbf{7 5 \%}$ \\
\hline
\end{tabular}

Recolección de datos: El instrumento aplicado medía, en una primera parte, variables sociolaborales y demográficas (establecimiento, grado académico, permanencia laboral en el establecimiento, grados académicos, capacitación, edad, entre otras) que permitieron caracterizar a la muestra. También se consultó la frecuencia de accidentes o eventos adversos para la salud como caídas, golpes, desmayos, convulsiones, hemorragias, fracturas, esguinces y vómitos, según la percepción de los funcionarios.

Una segunda parte contenía el instrumento "Percepción de la importancia de la enfermera escolar"(18), para medir, desde las perspectivas de los funcionarios, la importancia otorgada a las funciones asistenciales, investigativas, educativas, administrativas y de gestión de la enfermera escolar. Este es un cuestionario validado que consta de 29 ítems con cuatro opciones de respuesta, puntuadas en una escala tipo Likert, que va desde 1 (totalmente en desacuerdo) hasta 4 (totalmente de acuerdo).

Al ser este un estudio de opinión, no se estimó el riesgo de aparición de variables confusoras, siempre y cuando el instrumento fuera claro, preciso en su redacción y aplicado en un contexto adecuado. Debido a esto el instrumento fue evaluado por pares, educadores, enfermeros y otros profesionales de la salud, los cuales sugirieron correcciones al borrador inicial y a la versión final. Con el propósito de evitar sesgos de información, previó a la aplicación de la encuesta, se entregó información respecto de las funciones que cumple el profesional de enfermería en la promoción de la salud en personas sanas y enfermas.

No siendo estos ítems exclusivos para analizar cada una de las funciones señaladas, se identificaron cuáles de ellos resultaron más relevantes según las percepciones de los funcionarios.

Procesamientoyanálisis dedatos: Sedebedestacar que en este estudio no se presentaron variables confusoras, lo que permitió su objetividad para el análisis de los resultados. Mediante el programa SPSS V.21, se utilizó estadística descriptiva, donde las variables cualitativas se analizaron por medio de tablas de frecuencia, porcentaje y la prueba Chi-2 $\left(\mathrm{X}^{2}\right)$. Las variables cuantitativas se expresaron con media y desviación estándar.

\section{RESULTADOS}

Caracterización de los profesores y funcionarios administrativos: Predominaron las mujeres en un $78 \%$ y la edad promedio del grupo fue de 35,5 años $(\mathrm{DS}=11,24)$, entre un rango de edad que fluctuó entre los 20 y 76 años. El promedio de años de trabajo en el establecimiento fue de 4,36 años, y 24 años el mayor tiempo que una persona llevaba trabajando en su establecimiento. La Tabla 2 señala que la mayor participación fue del Colegio Ciudad Educativa (33,3\%), la mayoría $(64,3 \%)$ tenía el grado de Licenciado y un $61,1 \%$ no había recibido capacitación en primeros auxilios.

Percepción de frecuencia de accidentes/ incidentes: La Tabla 3 muestra quelas caídas y golpes son el accidente más frecuente, señalado por 122 participantes, quienes perciben, mayoritariamente $(73,3 \%)$, que su frecuencia es diaria. El vómito es el segundo incidente más frecuente, señalado por 106 participantes, con una percepción mayoritaria de ocurrencia semanal (40\%). 
Coincidente con lo anterior, el rol asistencial se señala, con un $90 \%$ de acuerdo, que es el más necesario a desarrollar por la enfermera escolar.

Percepción de la importancia de la Enfermera Escolar: Considerando el cambio de la escala Likert a una escala de porcentaje, siendo el 1 un 25\% (no importante), hasta el 4 un 100\% (Muy importante), en la Tabla 4 se detallan las acciones que podría realizar la enfermera en el ámbito escolar, puntuadas según percepción de importancia.

Se observa que el nivel de acuerdo o importancia es superior al $80 \%$ en todas las acciones. La acción percibida como más relevante es "Atender primeros auxilios", percibida con un $93,5 \%$ de importancia. La actividad percibida con menor importancia, aun cuando tiene un alto porcentaje (82,5\%), es la acción de realizar estudios científicos para conocer actitudes y prácticas inadecuadas en los estudiantes.

Tabla 2. Distribución de funcionarios según establecimientos educacionales, grado académico y capacitación en primeros auxilios, Sector Las Mariposas, Comuna de Chillán-Chile, 2018 ( $\mathrm{n}=126)$.

\begin{tabular}{llrr}
\hline Variables & Establecimiento & Fr & \% \\
\hline Establecimiento & Escuela Camilo Henríquez G-283 & 12 & 9,5 \\
& Escuela Especial de Lenguaje My College & 28 & 22,2 \\
& Colegio Adventista Las Mariposas & 25 & 19,8 \\
& Colegio Alcázares de Nuble & 19 & 15,0 \\
& Colegio Ciudad Educativa & 42 & 33,3 \\
\hline Grado académico & Licenciado & 81 & 64,3 \\
& Magíster & 5 & 3,9 \\
& Doctor & 1 & 0,8 \\
& Otro & 39 & 30,9 \\
\hline Capacitación en Primeros Auxilios & No & 77 & 61,1 \\
& Si & 49 & 38,8 \\
\hline
\end{tabular}

Tabla 3. Percepción de la frecuencia de accidentes en funcionarios de establecimientos educacionales, Sector Las Mariposas, Comuna de Chillán-Chile, 2018.

\begin{tabular}{|c|c|c|c|c|c|c|c|c|c|c|c|}
\hline \multirow{3}{*}{$\begin{array}{c}\text { Tipo de accidentes/ } \\
\text { incidentes }\end{array}$} & \multirow[t]{3}{*}{$\mathbf{N}^{\circ}$} & \multicolumn{2}{|c|}{ No ocurre } & \multicolumn{8}{|c|}{ Si ocurre } \\
\hline & & \multirow[t]{2}{*}{ Fr } & \multirow[t]{2}{*}{$\%$} & \multicolumn{2}{|c|}{ Diario } & \multicolumn{2}{|c|}{ Semanal } & \multicolumn{2}{|c|}{ Mensual } & \multicolumn{2}{|c|}{ Total } \\
\hline & & & & Fr & $\%$ & Fr & $\%$ & Fr & $\%$ & Fr & $\%$ \\
\hline Caídas y golpes & 124 & 2 & 1,6 & 91 & 73,4 & 21 & 16,9 & 10 & 8,1 & 122 & 98,4 \\
\hline Desmayos & 117 & 56 & 47,9 & 1 & 0,9 & 11 & 9,4 & 49 & 41,9 & 61 & 52,2 \\
\hline Convulsiones & 113 & 82 & 72,6 & 1 & 0,9 & 3 & 2,7 & 27 & 23,9 & 31 & 27,5 \\
\hline Hemorragias & 115 & 72 & 62,6 & 3 & 2,6 & 16 & 13,9 & 24 & 20,9 & 43 & 37,4 \\
\hline Fracturas & 112 & 52 & 46,4 & 3 & 2,7 & 6 & 5,4 & 51 & 45,5 & 60 & 53,6 \\
\hline Esguinces & 115 & 38 & 33,0 & 6 & 5,2 & 18 & 15,7 & 53 & 46,1 & 77 & 67,0 \\
\hline Vómitos & 120 & 14 & 11,7 & 16 & 13,3 & 48 & 40,0 & 42 & 35,0 & 106 & 88,3 \\
\hline
\end{tabular}

*A pesar de que $\mathrm{N}=126$, los ítems presentaron omiciones, por lo que $\mathrm{N}$ varía en cada tipo de accidente 
Tabla 4. Percepción de la importancia de la Enfermera Escolar según Acciones de enfermería a desarrollar en establecimientos educacionales, Sector Las Mariposas, Comuna de Chillán-Chile ( $\mathrm{n}=126)$.

\begin{tabular}{|c|c|c|}
\hline Acciones de enfermería & $\%$ & DS \\
\hline 1. Atender primeros auxilios. & 93,50 & 16,48 \\
\hline 2. Ser de ayuda en la atención de problemas respiratorios & 89,50 & 18,53 \\
\hline $\begin{array}{l}\text { 3. Brindar asesoría en prevención de enfermedades frecuentes en la edad escolar (obesidad, } \\
\text { asma, alergias). }\end{array}$ & 89,50 & 18,03 \\
\hline 4. Estar en la intervención y atención de fracturas. & 88,25 & 18,40 \\
\hline 5. Proporcionar cuidado en un contexto escolar. & 91,50 & 14,93 \\
\hline 6. Actuar ante emergencias o accidentes que surjan durante el horario escolar. & 94,00 & 13,28 \\
\hline 7. Coordinar con el centro de salud sobre el control de vacunación de los alumnos & 89,25 & 16,85 \\
\hline 8. Ser un referente de la salud, para las organizaciones involucradas en la salud escolar. & 89,50 & 16,88 \\
\hline 9. Coordinar con el Cesfam y/o centro de salud sobre el plan de salud escolar. & 89,25 & 15,68 \\
\hline $\begin{array}{l}\text { 10. Realizar estudios científicos para indagar conocimientos, actitudes y prácticas inadecuados } \\
\text { de los escolares. }\end{array}$ & 82,50 & 18,53 \\
\hline 11. Colaborar en grupos de investigación con el propósito de validar programas educativos. & 86,25 & 18,60 \\
\hline $\begin{array}{l}\text { 12. Ayudar a difundir los resultados de los estudiantes a través de revistas científicas o en el } \\
\text { periódico escolar. }\end{array}$ & 83,75 & 18,80 \\
\hline 13. Capacitar a los padres de familias sobre las enfermedades frecuentes. & 89,75 & 15,90 \\
\hline 14. Educar al alumnado sobre alimentación sana. & 88,50 & 16,63 \\
\hline 15. Educar al alumnado sobre la prevención de accidentes comunes. & 90,00 & 16,18 \\
\hline 16. Educar al alumnado sobre el cuidado del medio ambiental. & 83,50 & 18,50 \\
\hline 17. Educar al alumnado del nivel primario sobre hábitos saludables & 89,75 & 16,53 \\
\hline 18. Educar al alumnado sobre infecciones de transmisión sexual & 91,75 & 15,50 \\
\hline $\begin{array}{l}\text { 19. Realizar controles antropométricos (Talla, Peso) para valorar el índice de obesidad de los } \\
\text { alumnos(as). }\end{array}$ & 90,50 & 15,48 \\
\hline 20. Realizar seguimiento y monitoreo sobre la salud de los estudiantes & 85,50 & 20,63 \\
\hline 21. Educar para la prevención de hábitos de drogadicción y/o alcoholismo & 89,25 & 17,20 \\
\hline 22. Realizar evaluación primaria bucodental & 86,00 & 19,63 \\
\hline 23. Educar a los docentes sobre enfermedades más comunes en el contexto escolar. & 88,00 & 16,98 \\
\hline 24. Promover el ejercicio físico. & 84,00 & 19,35 \\
\hline 25. Realizar control y seguimiento a los alumnos con enfermedades crónicas. & 88,75 & 17,23 \\
\hline 26. Realizar charlas contra la violencia escolar/ familiar, consumo de drogas, entre otros. & 86,50 & 18,10 \\
\hline 27. Educar sobre la salud sexual y reproductiva (prevención del embarazo) & 90,00 & 16,48 \\
\hline $\begin{array}{l}\text { 28. Los padres se sentirían tranquilos al saber que en el colegio hay un profesional de salud } \\
\text { velando por la integridad de los escolares. }\end{array}$ & 92,25 & 15,65 \\
\hline 29. La enfermera escolar podría incentivar a recordar fechas conmemorativas. & 87,75 & 16,95 \\
\hline
\end{tabular}

\section{DISCUSIÓN}

Del total de los encuestados se observa que más de la mitad no ha tenido capacitación en primeros auxilios, por lo que no estarían preparados para brindar una primera atención de ese tipo al alumnado. Esto es similar a lo presentado en un estudio hecho en España en el que se observa que más de la mitad de los funcionarios no tienen conocimientos suficientes en primeros auxilios ${ }^{(20)}$.

Se pudo constatar que a pesar de que hay funcionarios con 24 años trabajando en los establecimientos, estos no han recibido capacitación en salud, y esta evidencia deja en claro 
que es necesario tener un cambio de paradigma respecto a cómo se desarrollan las acciones de salud en el sistema escolar, acciones que necesitan de la presencia de una enfermera escolar para implementar procesos integrales en la comunidad educativa, que apunten por sobre todo al bienestar físico, mental y social de adolescentes y jóvenes en los establecimientos educacionales. Este profesional trabaja en la prevención, protección, recuperación y rehabilitación de la salud, dando especial énfasis a la educación individual y grupal, así como a estimular la participación activa de la comunidad escolar ${ }^{(21)}$. Tal como se menciona en Vargas et al. ${ }^{(22)}$ "si desde pequeños tenemos acceso a la salud, incluyendo la escuela, la salud de la comunidad podría mejorar sustancialmente y esto haría posible que nuestras visitas al médico no sean tan seguidas".

Si generalmente cada establecimiento educacional está expuesto a que el alumnado pueda sufrir lesiones, sobre todo en edades más pequeñas y tal como lo señalan los funcionarios encuestados, el alumnado ha sufrido lesiones tales como: caídas, golpes, vómitos, esguinces, desmayos y hasta convulsiones, en menor proporción. Esto no solo ocurre en establecimientos chilenos, pues estudios en otras partes del mundo, han tenido los mismos resultados. Destaca uno similar realizado en Europa en el cual aparecen las caídas como el accidente escolar más frecuente ${ }^{(23)}$ y hacen necesario la presencia de un profesional idóneo para otorgar la atención y evitar complicaciones mayores ${ }^{(8)}$.

Así mismo, los funcionarios, con una aprobación de 93,5\%, destacan la necesaria labor de enfermería en las unidades educativas, para atender emergencias durante el horario de permanencia de los estudiantes, coincidiendo con lo señalado en Spina et al. ${ }^{(24)}$ uno de los tantos roles de la enfermería, dentro de un centro educativo, es brindar primeros auxilios y atender una emergencia. En países como los Estados Unidos, esto ya es una realidad y coincidiendo con la opinión manifestada por casi la totalidad de los funcionarios participantes del estudio, cada institución educacional cuenta con una o más enfermeras según la cantidad de estudiantes $^{(17,24)}$.

Coincidiendo con Nasser y Latorre ${ }^{(25)}$, en este estudio se dio un alto valor $(92,25 \%)$ a que los padres de familia se sentirían tranquilos al saber que el establecimiento cuenta con un profesional de salud que vela por la integridad de los escolares.
Así lo confirman los mismos apoderados cuando expresan que se sentirían mucho más seguros de enviar a sus hijos a estudiar sabiendo que la institución cuenta con la persona que posee la experticia para tratar, por ejemplo, a los niños con enfermedades crónicas, como la diabetes mellitus, quienes deben administrarse insulina con frecuencia ${ }^{(25)}$.

Por otro lado, una de las principales preocupaciones del Ministerio de Salud es el aumento de las enfermedades de transmisión sexual $^{(26)}$, preocupación que también se señala en este estudio cuando los funcionarios manifiestan un acuerdo del $91,75 \%$ respecto a que el alumnado debiera ser educado en esta materia, a temprana edad, por un profesional de enfermería, con énfasis en su prevención. El personal de enfermería es el agente efectivo para otorgar orientación y educar respecto a las enfermedades de transmisión sexual, especialmente las adolescentes, porque se identifican más fácilmente con las enfermeras que con otros miembros del equipo de salud, debido a factores relacionados con la identidad social, cultural y de género ${ }^{(27)}$.

Un último aspecto a considerar es que los funcionarios dan mucha importancia $(91,2 \%)$ al rol asistencial de la enfermera escolar, superando a los roles de administración, docencia e investigación. La enfermera escolar, en el ámbito asistencial individual, realiza atención inmediata a los alumnos afectados por algún incidente, maneja oportunamente las emergencias ocurridas durante el horario escolar, desarrolla planes de cuidados, controla a los alumnos con enfermedades crónicas $^{(28)}$ y, en el ámbito asistencial colectivo, identifica y valora las necesidades de salud del establecimiento educacional ${ }^{(16)}$.

Con respecto a la generalización de los resultados, el presente trabajo es evidencia de la necesidad de la incorporación de la enfermera escolar en los colegios básicos, y si bien la principal limitación del estudio corresponde al contexto evaluado: exclusivo de un escenario local de una ciudad al sur de Chile, se destaca que en el país se necesita más evidencia para visibilizar la necesidad de incorporar las competencias y habilidades propias del profesional de enfermería en el contexto educativo nacional.

Se deben realizar nuevas investigaciones que permitan observar la realidad de otras 
poblaciones escolares similares, identificando cuáles tareas asistenciales, administrativas, de investigación y docencia, relacionadas con la prevención y mantenimiento de la salud de los escolares, necesitan de un cuidado más directo y cuáles son las disparidades que, en este ámbito, surgen al comparar diferentes establecimientos educacionales.

\section{CONCLUSIÓN}

Según la percepción de los funcionarios encuestados en los colegios del sector, la presencia de un profesional del área de la salud, como la enfermera, es muy importante para realizar diversas funciones y la educación para la salud se observa

\section{REFERENCIAS}

1. Ministerio de Educación. Ley No 19.532 Crea el régimen de jornada escolar completa diurna y dicta normas para su aplicación [Internet]. Biblioteca del Congreso Nacional. 2006 [citado 20 ago 2019]. Disponible en: http://www.leychile. $\mathrm{cl} / \mathrm{n}$ ? $\mathrm{i}=88581 \& \mathrm{f}=2006-04-06 \& \mathrm{p}=$

2. Martín RA. Educación para la salud en primeros auxilios dirigida al personal docente del ámbito escolar. Enfermería Universitaria [Internet]. 2015 [citado 27 jun 2019]; 12(2): 88-92. Disponible en: http://www.scielo.org.mx/pdf/eu/v12n2/16657063-eu-12-02-00088.pdf

3. Palavecino T, Otárola D, Mihovilovic C. Caracterización de los accidentes escolares atendidos en el servicio de urgencia del hospital Roberto del Río en el ańo 2005. Rev Ped Elec [Internet]. 2009 [citado 27 jun 2019]; 6(2): 17-32. Disponible en: http://www.revistapediatria.cl/volumenes/2009/ vol6num2/pdf/CARACTERIZACION.pdf

4. Vásquez A, Astorga M, Sanhueza P. Enfermería. Colegio del Verbo Divino [Internet]. 25 sep 2015 [citado 27 jun 2019]. Disponible en: https://www. cvd.cl/enfermeria/

5. Colegio San Agustín. Enfermería. Colegio San Agustín [Internet]. 22 jun 2014 [citado 27 jun 2019]. Disponible en: https://www. colegiosanagustin.cl/enfermeria/

6. Colegio Suizo de Santiago. Enfermería Informativo [Internet]. Santiago: Colegio Suizo de Santiago. [citado 27 jun 2019]. Disponible en: http://www. css.cl/desarrollo/aula-virtual como un punto importante a valorar dentro de los establecimientos educacionales.

Los resultados señalan que tener una enfermera escolar permite enfrentar problemáticas mediante acciones de promoción y prevención de la salud realizando controles de salud escolar, vacunación, educación sobre nutrición, sexualidad e higiene, como también prevención y atención de accidentes dentro del establecimiento.

Esta visión no solo es de los funcionarios pues, a opinión de ellos, los padres y sus familias también la consideran un recurso vital que debe estar presente en los establecimientos educacionales, cuyo valor está en el trabajo integrado que puede realizar en la comunidad escolar, en coordinación con los profesionales de los centros de salud primarios.

7. Encinar A. Enfermería escolar, la situación hoy en día. Rev enferm CyL [Internet]. 2015 [citado 27 jun 2019]; 7(1): 56-61. Disponible en: http:// www.revistaenfermeriacyl.com/index.php/ revistaenfermeriacyl/article/view/144

8. Araujo P. Importancia del rol de la enfermera escolar en los centros educativos de las islas canarias. Rev ROL Enf [Internet]. 2013 [citado 27 jun 2019]; 36(7-8): 36-42. Disponible en: https://dialnet. unirioja.es/servlet/articulo?codigo $=4401017$

9. Mori F, Bustamante S, Leitón Z, Santillan R. Competencias de la enfermera en instituciones educativas: una mirada desde los gestores educativos. Rev Gaúcha Enferm [Internet]. 2018 [citado 3 feb 2020]; 39: e2017-0152. Disponible en: http://www.scielo.br/scielo.php?script=sci arttext\&pid=S1983-14472018000100432

10. Colegio Instituto San José. Protocolo de enfermería y accidentes escolares [Internet]. Cañete, Chile: Colegio Instituto San José [citado 27 jun 2019]. Disponible en: http://www.sanjo.cl/web/wpcontent/uploads/2013/10/PROTOCOLODE - EN FER MERIA-Y-ACCIDENTES ESCOLARES-SANJO.pdf

11. Colegio Leonardo Da Vinci. Protocolo enfermería escolar Colegio Leonardo Da Vinci de Las Condes [Internet]. Santiago, Chile: Colegio Leonardo Da Vinci de Las Condes [citado 30 jun 2019]. Disponible en: https://www.corplascondes.cl/ contenidos/transparencia/ley_de_transparencia/ tramites-requisitos/educacion/leonardo_davinci/ Protocolos/PROTOCOLO_ENFERMERIA_ ESCOLAR_2014.pdf 
12. Colegio Britanico Saint Margarets S.A. Protocolo atención enfermería [Internet]. Enfermería. Concón: Colegio Britanico Saint Margarets S.A. 2014 [citado 3 feb 2020]. Disponible en: http:// www.stmargarets.cl/info/protocolo_enfermeria.pdf

13. Colegio Instituto San José. Manual de sala de enfermería [Internet]. Cañete, Chile: Colegio Instituto San José [citado 30 jun 2019]. Disponible en: http://www.sanjo.cl/web/wpcontent/uploads/2017/12/Protocolo-de-uso-sala-deenfermer\%C3\%ADa-ISJ.pdf

14. Sociedad Chilena de Enfermería en Salud Escolar. Misión y visión [Internet]. Santiago, Chile: Sociedad Chilena de Enfermería en Salud Escolar [citado 30 jun 2019]. Disponible en: http://www. sochiese.cl/mision-y-vision

15. García K, Mejía B, Rivera O. Efectividad de una intervención educativa para la promoción de alimentación saludable en niños en etapa escolar [Tesis de Especialidad en Enfermería en Salud Familiar y Comunitaria]. Lima (Perú): Universidad Norbert Wiener; 2018. Disponible en: http://repositorio.uwiener.edu.pe/bitstream/ handle/123456789/2036/ESPECIALIDAD\%20 -\%20 Garcia\%20Pinedo\%2C\%20Katherine. pdf?sequence $=1 \&$ isAllowed $=y$

16. De Arco-Canoles O, Suárez-Calle Z. Rol de los profesionales de enfermería en el sistema de salud colombiano. Univ Salud. 2018; 20(2): 171-182. Disponible en: http://www.scielo.org.co/pdf/reus/ v20n2/0124-7107-reus-20-02-00171.pdf

17. Zabalequi A. El rol del profesional en enfermería. Aquichan. 2003; 3(1): 16-20. Disponible en: http://www.scielo.org.co/scielo.php?script=sci_ abstract\&pid=S1657-59972003000100004

18. Rojas GI, Vargas CI, Ferrer IL. Investigaciones Rol de enfermería en la limitación del esfuerzo terapéutico en el paciente crítico. Cienc enferm. 2013; 19(3): 41-50. Disponible en https://scielo.conicyt.cl/scielo.php?script=sci_ arttext\&pid=S0717-95532013000300005

19. García A. Manual de primeros auxilios para docentes [Internet]: American Academy [citado 30 jun 2019]. Disponible en: https://www.academia. edu/27377660/MANUAL_DE_PRIMEROS_ AUXILIOS_PARA_DOCENTES

20. Velasco Z, Gaintza Z. Análisis del grado de formación en primeros auxilios del profesorado en activo de educación infantil y primaria. Formación universitaria [Internet]. 2017 [citado 3 feb 2020]; 10(2): 67-68. Disponible en: https://scielo.conicyt. $\mathrm{cl} / \mathrm{pdf} /$ formuniv/v10n2/art08.pdf

21. Colegio de Enfermeras de Chile. Codigo de ética [Internet]. Santiago, Chile: Colegio de Enfermeras de Chile: 2008 [citado 30 jun 2019]. Disponible en: http://www.bioeticachile.cl/felaibe/documentos/ chile/Codigo\%20Etica\%20Enfermeria\%20Chile. PDF

22. Vargas I, Villegas O, Sánchez A, Holthuis K. Promoción, Prevención y Educación para la Salud. Intendencia de Montevideo [Internet] 2003 [citado 3 feb 2020]. Disponible en https://montevideo. gub.uy/sites/default/files/concurso/documentos/ Ed211.paralaSalud-MariaT.Cerqueira.pdf

23. Onís GE, Varona PI, Gil PM, Felici C, Embid P. Lesiones no intencionadas en el centro escolar: ¿de qué estamos hablando? Rev Pediatr Aten [Internet]. 2015 [citado 3 feb 2020]; 17(68): 333-339. Disponible en: http://scielo.isciii.es/scielo.php?script=sci arttext\&pid=S1139-76322015000500008

24. Spina R, Jara F, Rodríguez S. La importancia de la figura enfermera en un centro escolar. Rev electrón PortalesMédicos.com [Internet]. 2017 [citado 3 feb 2020]. Disponible en: https://www.revistaportalesmedicos.com/revista-medica/enfermeracentro-escolar/

25. Nasser O, Latorre I. La enfermería escolar: Una necesidad sentida [Tesis de Maestría]. Almería (Espańa): Universidad de Almería; 2011. Disponible en: https://docplayer.es/7653848-Laenfermeria-escolar-una-necesidad-sentida.html

26. Salas F, Partarrieu S. Las ITS en adolescentes: ¿Qué nos dicen las cifras?. El estetoscopio [Internet]. 2018 [citado 4 de jul 2019]; 96 (1): 7-10. Disponible en: https://www.sochipe.cl/subidos/ revista1/docs/EE\%20N96\%20-\%20FINAL.pdf

27. Sánchez B, Hernández M. Conocimiento y aprendizaje de enfermeras(os) sobre infecciones de transmisión sexual. Perinatol Reprod Hum [Internet]. 2005 [citado 4 de jul 2019]; 19(3-4): 177-186. Disponible en: http://www.scielo.org. mx/pdf/prh/v19n3-4/v19n3-4a7.pdf

28. Ibarra A. Preocupa escasez de enfermeras escolares. La opinión [Internet]. 08 may 2018 [citado 4 jul 2019]. Disponible en: https:// laopinion.com/2018/05/08/preocupa-escasez-deenfermeras-escolares/ 